\title{
Testing operational phase concepts in quantum optics
}

\author{
Jaroslav Řeháček $\dagger$, Zdeněk Hradil $\dagger$, Miloslav Dušek $\dagger$, \\ Ondřej Haderka $\uparrow+$ and Martin Hendrych $\dagger \neq$
}

$\dagger$ Department of Optics, Palacký University, 17. listopadu 50, 77200 Olomouc, Czech

Republic

† Joint Laboratory of Optics of Palacký University and Physics Institute of the Czech

Academy of Science, 17. listopadu 50, 77200 Olomouc, Czech Republic

Received 31 January 2000, in final form 15 March 2000

\begin{abstract}
An experimental comparison of several operational phase concepts is presented. In particular, it is shown that statistically motivated evaluation of experimental data may lead to a significant improvement in phase fitting upon the conventional procedure of Noh et al (1993 Phys. Rev. Lett. 71 2579). The analysis is extended to the asymptotic limit of large intensities, where a strong evidence in favour of multi-dimensional estimation procedures has been found.
\end{abstract}

Keywords: Phase estimation, interferometry, quantum phase, state reconstruction

\section{Introduction}

'The essence of quantum theory is its ability to predict probabilities for the outcomes of tests, following specified preparations' [1]. From a pragmatic point of view the quantum state represents only our information on the system corresponding to a particular preparation by a classical apparatus. According to quantum theory, this seems to be the most complete information. However, the accessibility of this information is questionable. Not knowing the preparation procedure, one does not know the quantum state of the system. There is no way to measure it for a single realization of a quantum system. The situation gets better if an ensemble of systems prepared in the same quantum state is available. Then it is possible to measure complementary observables in different experiments, and the quantum state of the system can be inferred. Since real ensembles are always finite, only the particular numbers of occurrence of different results can be measured instead of probabilities. This is a paradigm for an arbitrary measurement. However, this scheme could seem purposeless unless theoretical predictions are compared with experiments. In the quantum domain this is generally not at all easy, and in practice many sophisticated theories cannot be demonstrated on their experimental counterparts.

The estimation of phase differences in interferometry appears to be a nice example of the above-mentioned scheme, where the predictions of quantum theory can be followed by an experimental realization. Optical measurements in the domain of classical wave optics are well established and belong to the most precise measurement schemes currently available. Significantly, such schemes may be analysed in the framework of quantum phase.
Quantization based on the correspondence principle leads to the formulation of operational quantum phase concepts [2,3]. Further generalization may be given in the framework of quantum estimation theory; the prediction may be improved using the maximum-likelihood (ML) estimation. This improvement was tested experimentally in matter wave optics with neutrons, and a statistically significant improvement was observed [4]. This is a remarkable result, since the phase estimation is rather uncertain for neutrons due to technical limitations of neutron interferometry; for example, where the visibility of interference fringes is far below the ultimate value of $100 \%$. In this paper the same theoretical background of optimal phase estimation will be used for the testing of phase resolution with photons.

Optical measurements offer many advantages. Current optical technology enables us to achieve visibility of interference fringes close to unity, and to very precisely set the intensities of light pulses at levels well below one photon on average. As the main objective, different strategies for accurate phase estimation will be specified and their consequences for achieved precision will be derived.

This paper is organized as follows. The mathematical tools are reviewed in section 2, where the operational phase concepts are naturally embedded in the quantum estimation theory. The experimental set-up is described in section 3 . A comparison of several phase estimation procedures based on the experimentally measured data is given in section 4 . Finally, section 5 deals with the phase estimation in the asymptotic regime. 


\section{Phase estimation}

The operational phase concepts can naturally be embedded in the general scheme of quantum estimation theory $[5,6]$ as in $[4,7,8]$. Let us consider the eight-port homodyne detection scheme [2,9] with four output channels numbered by indices 3, 4, 5 and 6, where the actual values of intensities are registered in each run. Assume that these values fluctuate in accordance with some statistics. The mean intensities are modulated by a phase parameter $\bar{\theta}$

$$
\begin{aligned}
& \bar{n}_{3,4}=\frac{N}{2}(1 \pm V \cos \bar{\theta}), \\
& \bar{n}_{5,6}=\frac{N}{2}(1 \pm V \sin \bar{\theta}),
\end{aligned}
$$

where $N$ is the total intensity and $V$ is the visibility of interference fringes. The true phase shift inside the interferometer $\bar{\theta}$, which is a nonfluctuating parameter controlled by the researcher, should carefully be distinguished from the estimated phase shift, which is a random quantity. Hereafter, the latter is denoted by $\theta$. This device, if operated with Gaussian signals, represents nothing but a classical wave picture of the original eight-port homodyne detection scheme. Equivalently, it also corresponds to a Mach-Zehnder interferometer, when the measurement is performed with zero and $\pi / 2$ auxiliary phase shifters. In this case, data is not obtained simultaneously, but is collected during repeated experiments. Providing that a particular combination of outputs $\left\{n_{3}, n_{4}, n_{5}, n_{6}\right\}$ has been registered, the phase shift can be inferred. The point estimators of phase corresponding to the ML estimation will be used here [10,11]. In accordance with the ML approach [12], the sought-after phase shift is given by the value, which maximizes the likelihood function. Provided that the noise is Gaussian, the likelihood function corresponding to the detection of given data readings

$$
\mathcal{L} \propto \exp \left\{-\frac{1}{2 \sigma^{2}} \sum_{i=3}^{6}\left[n_{i}-\bar{n}_{i}\right]^{2}\right\} .
$$

Here the variance $\sigma^{2}$ represents the phase insensitive noise of each channel. A notation analogous to the definition of phase by Noh et al [3] (NFM) may be introduced:

$$
\begin{aligned}
\mathrm{e}^{\mathrm{i} \theta_{\mathrm{NFM}}} & =\frac{n_{3}-n_{4}+\mathrm{i}\left(n_{5}-n_{6}\right)}{\sqrt{\left(n_{3}-n_{4}\right)^{2}+\left(n_{5}-n_{6}\right)^{2}}}, \\
V^{\prime} & =\sqrt{\left(n_{3}-n_{4}\right)^{2}+\left(n_{5}-n_{6}\right)^{2}} .
\end{aligned}
$$

The likelihood function (2) may be maximized by the choice of parameters for phase shift and visibility, respectively [4]

$$
\begin{gathered}
\theta=\theta_{\mathrm{NFM}}, \\
V=\min \left(\frac{2 V^{\prime}}{\sum_{i=3}^{6} n_{i}}, 1\right) .
\end{gathered}
$$

Hence, the operational phase concept of Noh et al [3] coincides with the ML estimation for waves represented by the continuous Gaussian signal with phase independent and symmetrical noises. These rather strict assumptions are incompatible with the nature of signals encountered in experiments; such restrictions would be, however, natural in the classical theory.

The optimum prediction is different for Poissonian statistics. Based on the Poissonian likelihood function

$$
\mathcal{L} \propto \prod_{i=3}^{6} \bar{n}_{i}^{n_{i}}
$$

ML estimation gives optimum values for phase shift and visibility [4]

$$
\begin{gathered}
\mathrm{e}^{\mathrm{i} \theta}=\frac{1}{V}\left[\frac{n_{4}-n_{3}}{n_{4}+n_{3}}+\mathrm{i} \frac{n_{6}-n_{5}}{n_{6}+n_{5}}\right], \\
V=\sqrt{\left(\frac{n_{4}-n_{3}}{n_{4}+n_{3}}\right)^{2}+\left(\frac{n_{6}-n_{5}}{n_{6}+n_{5}}\right)^{2}},
\end{gathered}
$$

provided the estimated visibility (9) is smaller than unity. In the opposite case it is necessary to maximize the likelihood function (7) on the boundary $(V=1)$ of the physically allowed region of the parameter space numerically. Relations (8) and (9) provide a correction of the Gaussian theory with respect to the discrete signals. Besides the phase shift, visibility of interference fringes and the total input intensity can be evaluated simultaneously.

The apparent difference between relations (5) and (6) and (8) and (9) represents the theoretical background of the presented treatment. Obviously, both predictions will coincide provided that there is almost no information available in the low-field limit $N \rightarrow 0$. Similarly in the strong-field limit $N \rightarrow \infty$, the phase of the light is well defined and both inferred values of the phase approach the same value. Possible deviations may appear in the intermediate regime $N \approx 1$. The test of the difference between (5) and (8) is proposed through controlled phase measurement. The phase difference was adjusted to a certain value and estimated independently using both methods (5) and (8) in repeated experiments.

To compare two or more phase estimators, some measure of the estimation error is needed. Dispersion, defined as

$$
\sigma^{2}=1-\left|\left\langle\mathrm{e}^{\mathrm{i} \theta}\right\rangle\right|^{2}
$$

can do the job well. Here, the average is taken over posterior phase distribution of the corresponding phase estimator. The dispersion (10) is a compact space analogy of the averaged quadratic cost function (variance), frequently used in estimation theory [5].

The evaluation of the average quadratic cost (10) is not the only way to compare efficiencies of different estimation procedures. Another possibility is to use the rectangular cost function

$$
C(\theta-\bar{\theta})= \begin{cases}-1 & |\theta-\bar{\theta}| \leqslant \Delta \theta \\ 0 & |\theta-\bar{\theta}|>\Delta \theta\end{cases}
$$

This choice of the cost function corresponds to the following evaluation the experimental data. Each sample of data consisting of numbers $n_{3}, n_{4}, n_{5}$ and $n_{6}$ of counted photons is processed using the NFM formula (3) issuing phase prediction $\theta_{\mathrm{NFM}}$. The relative frequency $f_{\mathrm{g}}(\Delta \theta)$, which is 
proportional to the average cost of the Gaussian estimator $\langle C(\theta-\bar{\theta})\rangle$, characterizes how many times the estimated phase $\theta_{\mathrm{NFM}}$ falls within the chosen phase window $\Delta \theta$ (confidence interval) spanning around the true phase shift. The same procedure is repeated for phase predictions based on the Poissonian phase estimator (8) yielding the relative frequency of 'hits' $f_{\mathrm{p}}(\Delta \theta)$. The quantity

$$
\Delta E=f_{\mathrm{P}}(\Delta \theta)-f_{\mathrm{G}}(\Delta \theta)
$$

represents the difference in efficiency of the ML and NFM phase estimations for the given phase window $\Delta \theta$ and given input intensity $N$. If this quantity is significantly positive, the ML estimation is better than its NFM counterpart. On the other hand, if $\Delta E$ is close to zero, both data evaluation procedures are statistically equivalent and no discrimination is possible.

\section{Experimental set-up}

The laboratory set-up (see figure 1) is based on a singlemode-fibre Mach-Zehnder interferometer carefully balanced and adjusted for maximum visibility. A semiconductor laser source (SHARP LT015) produces 4 ns long pulses with a repetition rate of $130 \mathrm{kHz}$. The initial pulse intensity is about $10^{7}$ photons per pulse. This is decreased by $11 \mathrm{~dB}$ due to losses in the fibres and other components of the set-up, and precisely adjusted by artificial attenuation in the programmable attenuator (JDS Fitel HA9) to reach the required level at the detectors. The input coupler FC (SIFAM) divides the pulses between the arms of the interferometer (each $4 \mathrm{~m}$ long). Both arms contain planar phase modulators PM1,2 (UTP). Only PM1 has been used for phase settings, the other is included just for symmetry reasons. Both modulators also work as linear polarizers (extinction ratio $1: 10^{6}$ ) to improve the degree of polarization. Input polarization to the modulators is set by polarization controllers PC1 and PC2. Attenuator ATT in the upper arm of the interferometer helps balance the losses in both arms of the interferometer to reach maximum visibility. The length of the arms is balanced by a variable air gap (AG). The polarization controller PC3 is used to match the polarization in the arms at the output variable ratio coupler SVRC (SIFAM). The resultant interference is detected using silicon photon-counting detectors (EG\&G SPCM-AQ) with less than 70 dark counts per second and a quantum efficiency of $55 \%$. The signals from the detectors are processed using detection electronics based on time-toamplitude converters and single-channel analysers (EG\&G Ortec) and recorded by a computer, which also controls the driving voltage of the phase modulator, programmable attenuator setting and laser operation as well. In this set-up we have reached interference visibility of up to $99.8 \%$.

The whole interferometer is placed in a polystyrene box to minimize thermal drift of the fringes. After initial warm-up, the phase stability of the device is better than $\pi / 3000 \mathrm{~s}^{-1}$. During the measurement, active stabilization of the interference pattern is performed each 5-10 s.

\section{Measured data evaluation}

Unfortunately, commercially available photodetectors for measurement of weak quantum signals fail to discriminate the number of detected photons. Only the presence or absence of the signal can usually be detected. The impossibility of counting photons is circumvented as follows. According to the well known polynomial theorem, the sum of two or more Poissonian signals is a Poissonian signal again, the mean simply being replaced by the sum of the means of its constituents. It is therefore possible to carry out measurements with very weak signals of intensity, say, $0.01-0.001$ photons per pulse so that the probability of two photons being in the same pulse (double-detection) is very small, and then collect an appropriate number of individual yes-no detections to obtain desired 'input' intensity $N$. For example, an experimental run with input pulse mean intensity $N=10$ can be simulated by a sequence of 10000 measurement with mean input intensity $N_{p}=0.001$ photons per pulse. The probability of double-detection in a single run is $p<10^{-6}$ for a Poissonian light source. Hence the probability of single double-detection during the whole sequence of measurements is less than $1 \%$ and the probability of triple-detection or several double-detections is entirely negligible. This procedure enables us to effectively simulate the results of experiments with intense pulses $N \gg 1$ and ideal photodetectors. In the text, whenever an experimental sample is mentioned, it should be clear that we actually refer to a sum of many experimental samples measured with intensities well below a single-photon per pulse.

The difference of dispersions (10) of the Gaussian and Poissonian phase estimators found in our experiment is shown in figure 2 for a fixed true phase $\bar{\theta}=\pi / 3$. The number of experimental samples used for calculation of the dispersions varies from 1000 samples for input intensity $N=60$ to more than 100000 samples with $N=0.1$. The error bars arising from a limited number of samples are the result of numerical simulation. Figure 2 agrees well with the qualitative reasoning of the previous section. The most distinct difference between the dispersions of the ML and NFM estimators is seen for the input mean number of photons $N \approx 7.5$. Thus, it can be said that as long as interference and phase measurements are concerned, discrete signals with Poissonian statistics are distinguishable from the classical wave for only a relatively small range of input intensities.

The difference in efficiency of the ML and NFM phase estimation (12) calculated from experimental data is shown in figure 3. The difference $\Delta E$ was calculated using 7500 experimental samples measured in experiment with $N=10$ photons and visibility of $99.6 \%$. The chosen input intensity roughly corresponds to the maximum seen in figure 2 . Since the experimental data are limited to a finite number of samples due to experimental conditions and available time, the estimated $\Delta E$ would be slightly different in repeated experiments. Statistical significance of the experimental results is demonstrated using computer simulation again. Standard deviation corresponding to 7500 measured samples is shown in figure 3 as error bars for each phase window.

A significant difference between the effectiveness of classical and optimal treatments is apparent in figure 3. The 


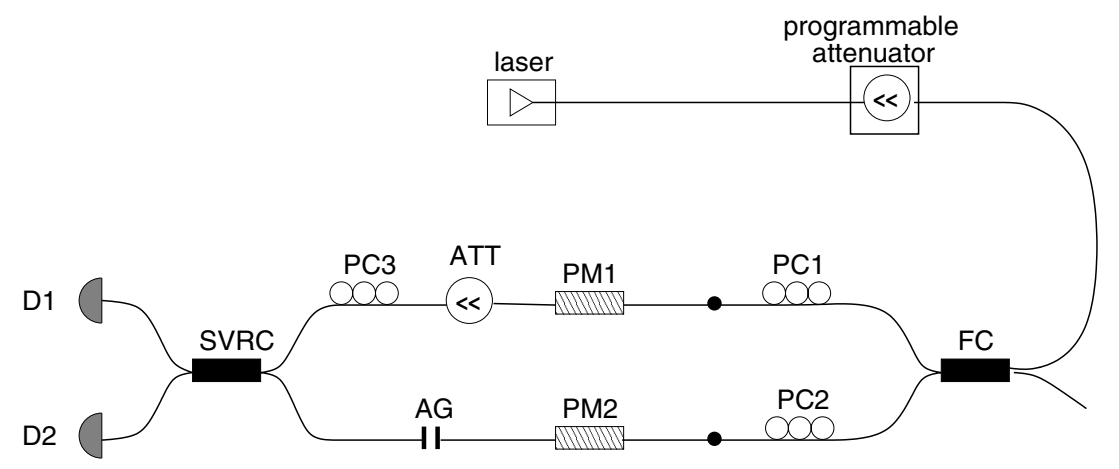

Figure 1. Scheme of the laboratory set-up. FC, input fibre coupler; PC $x$, polarization controllers; PM $x$, phase modulators; ATT, attenuator; SVRC, output variable ratio coupler; D $x$, detectors.

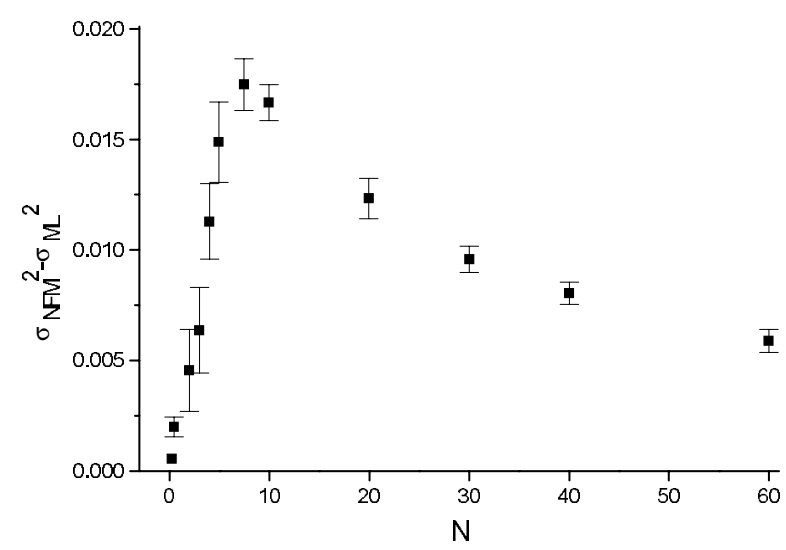

Figure 2. The experimentally observed difference between dispersions of the NFM and ML estimators as a function of the input mean number of photons $N$ for fixed true phase $\bar{\theta}=\pi / 3$. Error bars corresponding to $68 \%$ confidence intervals are shown.

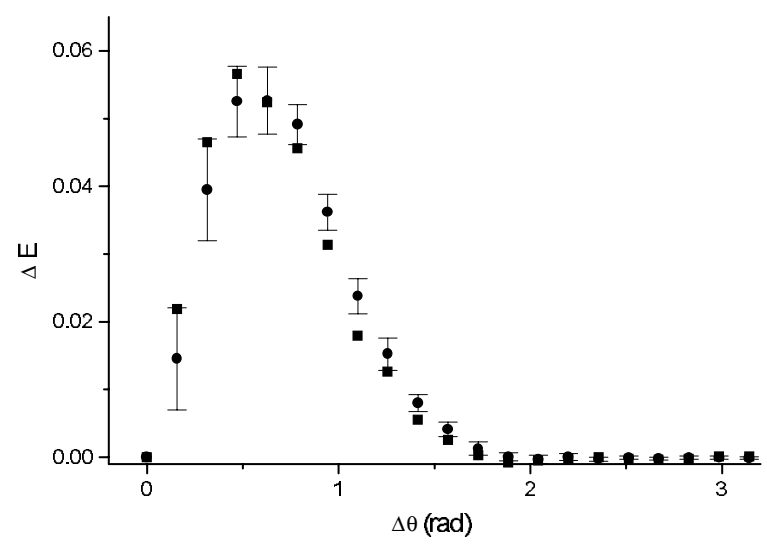

Figure 3. Experimentally obtained $\Delta E$ (squares) compared with theoretical values (circles); for the definition of $\Delta E$, see equation (12); $\Delta \theta$ is the phase window. Error bars corresponding to 7500 measured samples are shown.

optimal treatment provides an improvement in estimation procedure, and the difference is beyond the statistical error by more than 10 standard deviations in the optimum case. High stability and visibility of interference fringes in the optical interferometer along with a high repetition rate of the pulsed laser make the improvement over the NFM phase prediction more evident than in a similar comparison performed with the neutron interferometer set-up [4]. Notice the dependence of the precision gain $\Delta E$ on the width of the chosen phase window. Obviously, no better performance of the ML method can be expected for large values of the phase window $\Delta \theta$; any sensible statistical method would yield quite reasonable results. Likewise, no real improvement over the Gaussian estimate can be expected when $\Delta \theta$ is close to zero, because too few data would then fall within the window. The largest difference is about $6 \%$ in the window of the width of about $0.5 \mathrm{rad}$.

\section{Asymptotic behaviour}

As intensity of the input light increases, both estimations of phase shift yield more sharp and precise results. The error of the best-known proposed phase measurements scales as $N^{-1}$ for large input intensities $[13,14]$. Our photodetection scheme cannot compete with such measurements. On the other hand these methods necessitate the use of exotic states such as two-mode Fock states, etc, which are still impossible to prepare in contemporary laboratories. Since the experimental equipment is always limited, the only way to improve precision of phase measurements lies in careful evaluation of the measured phase sensitive data and distillation of all available phase information. Therefore, it is worthwhile to compare the performance of the NFM and ML estimations in the limit of high intensities.

To get some qualitative feeling of how both estimators approach the above mentioned limit we redrew figure 2 with differently scaled vertical axes, see figure 4 . It can be seen that the relative difference of both dispersions monotonically increases with $N$ and finally approaches some constant value different from zero. This means that during the transition from $N \approx 10$ to higher values of $N$, both estimators first scale with slightly different powers of $N$, and for high intensities both powers reach the same value and the ratio of the Gaussian and Poissonian dispersions approach some constant.

It is easy to calculate the behaviour of the dispersion of the Gaussian phase estimator for $N \rightarrow \infty$. The dispersion becomes

$$
\sigma_{G}^{2} \approx \frac{1}{V^{2}} N^{-1}+\mathrm{O}\left(\frac{1}{N^{2}}\right) .
$$

Readers interested in details are referred to appendix A. As can be expected, the error of NFM phase measurement is 


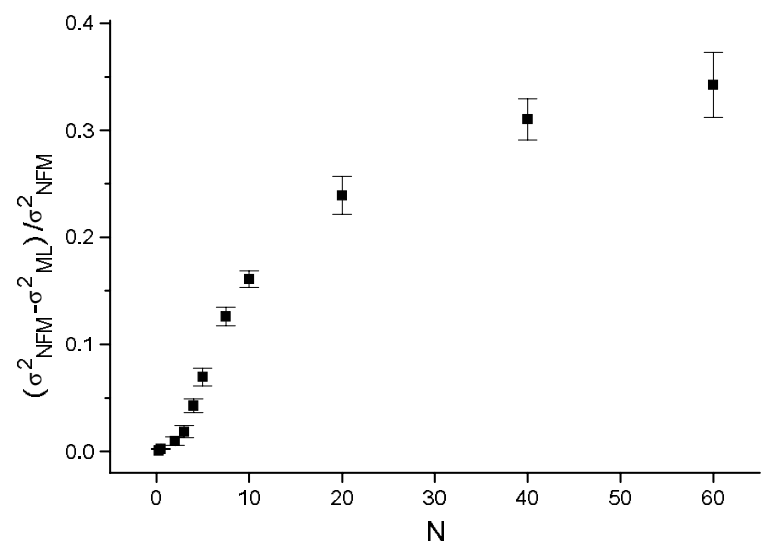

Figure 4. Observed relative difference between dispersions of the NFM and ML estimators. All the parameters are the same as in figure 2 .

proportional to $N^{-1 / 2}$. This precision represents the socalled standard quantum limit. Unfortunately, it is impossible to derive a simple expression similar to equation (13) for the Poissonian estimator. However, such a formula is easily obtained provided that the physical constraint $V \leqslant 1$ is released. Thus, throwing the estimated value of visibility away and interpreting equation (8) as an estimator of the unknown phase shift valid for each sample $\left\{n_{3}, n_{4}, n_{5}, n_{6}\right\}$, the asymptotic dispersion of such an unconstrained estimation reads

$$
\sigma_{P}^{2} \approx \frac{1}{V^{2}}\left(1-\frac{V^{2}}{2} \sin ^{2} 2 \bar{\theta}\right) N^{-1}+\mathrm{O}\left(\frac{1}{N^{2}}\right) .
$$

It is obvious that omitting useful information gained from the data makes the unconstrained estimation somewhat less efficient than the original constrained one.

We can see from equations (13) and (14) that, in the limit of low visibility, both the NFM and ML phase predictions are equivalent. This result is in agreement with properties of the well known discrete Fourier transform (DFT) phase estimator [15] in the same regime $\dagger$. On the other hand, ML estimation always gives better results than NFM theory, provided that the visibility is high. For some values of the true phase shift, reduction in the dispersion down to $50 \%$ is possible.

The ultimate limit to the resolution of the particular estimator is set by the well known Cramér-Rao inequality. Provided that the visibility and input intensity are under control in the experiment, the phase shift $\bar{\theta}$ remains the only parameter to be estimated. For such a single-parameter problem, the Cramér-Rao lower bound (CRLB) on the dispersion $\ddagger$ of any phase estimator is given as follows

$$
\sigma_{\mathrm{CRLB}}^{2}=\left(E\left\{\left[(\partial / \partial \bar{\theta}) \ln p\left(n_{3}, n_{4}, n_{5}, n_{6} \mid \bar{\theta}\right)\right]^{2}\right\}\right)^{-1} .
$$

Here the symbol $E$ denotes averaging over observed data. Upon substitution of the joint Poissonian distribution of the

$\dagger$ We note in passing that DFT can in fact be regarded as a generalization of NFM phase concept to a greater number of auxiliary phase shifts [4].

$\ddagger$ Actually, the CRLB holds for variance rather than for dispersion. Since all the relevant phase uncertainties are small in the limit of high input intensity, both quantities coincide in this case. sample $\left\{n_{3}, n_{4}, n_{5}, n_{6}\right\}$ to equation (15) and making similar approximations to those used in derivation of equation (13), we end up with

$$
\sigma_{\text {CRLB }}^{2}=\frac{V^{2}-1-\frac{1}{4} V^{4} \sin ^{2} 2 \bar{\theta}}{V^{2}-1-\frac{1}{2} V^{2} \sin ^{2} 2 \bar{\theta}} V^{-2} N^{-1} .
$$

Two interesting observations follow from (16). First, notice that for low visibility, the dispersion of the NFM phase prediction (13) attains the CRLB. This means the NFM estimation is best possible in this limit. Second, for perfect experimental set-up ( $V=1)$, the CRLB is simply $\sigma_{\text {CRLB }}^{2}=1 / 2 N$. Thus the resolution of the unconstrained ML estimation can still be improved a bit. In deriving equation (16) we supposed that the value of visibility and input intensity of the laser beam are known. Such knowledge represents some additional information about the experimental set-up. Let us assume that the input intensity and visibility are really under control and, in addition, the visibility is equal to unity. In this case the Poissonian likelihood function (7) depends only on the value of the phase shift $\bar{\theta}$. Now the single-parameter ML estimation of the phase shift $\bar{\theta}$ consists of maximizing the likelihood function $\mathcal{L}(\bar{\theta}, V=1)$ with respect to the single parameter $\bar{\theta}$. This procedure is just what we have done in the case of the many-parameter ML estimation equations (8) and (9), when the experimental sample yielded an unphysical value of visibility $V>1$. The only difference is that in the case of the single-parameter ML estimation we maximize the likelihood function on the boundary for any detected sample $\left\{n_{3}, n_{4}, n_{5}, n_{6}\right\}$. We may ask whether the single-parameter phase estimator achieves the best phase resolution $\sigma_{\mathrm{CRLB}}^{2}=$ $1 / 2 N$. An explicit calculation (for details see appendix B) shows this is really the case. Although it may seem that the single-parameter ML estimation thus gives best results, some caution is necessary when visibility (or another parameter) is not known precisely or fluctuates. For example, estimation on the boundary $V=1$ leads to a strongly biased phase prediction provided the actual visibility differs from unity, as is demonstrated in figure 5. In this particular case, the bias caused by dismissing the possibility $V<1$ is independent of the input light intensity $N$. For larger intensities it dominates the uncertainty of estimated phase and the single-parameter ML estimation may be outperformed by the Gaussian (NFM) one. Therefore, one should always estimate all parameters, which are not under the researcher's control together with the parameter of interest regardless of smaller theoretical effectiveness of such a complex estimation procedure.

Now let us return to the many-parameter constrained ML estimation. In this case a particular detection $\left\{n_{3}, n_{4}, n_{5}\right.$, $\left.n_{6}\right\}$ is processed either by equation (8) (when applied to all samples, $\sigma^{2}$ is given by equation (14)) or via maximization of likelihood function (7) on the boundary (when applied to all samples, $\left.\sigma^{2}=1 / 2 N\right)$. Although we do not switch between these methods at random, because the choice depends on the particular sample, the mean of both dispersions gives us a rough estimate of the performance of the constrained ML phase prediction. A more precise value can always be obtained with the help of computer simulation. The performances of various phase estimators are summarized in table 1 for a perfect experimental set-up $V=1$. 


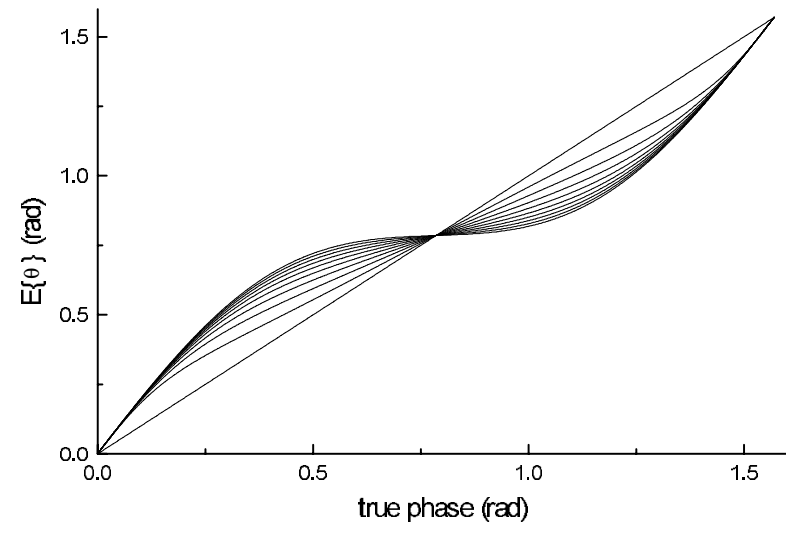

Figure 5. Bias of the single-parameter phase estimator for expected visibility $V=1$ as a function of the true phase shift $\bar{\theta}$. Actual visibility of interference fringes varies from $V=1$ (straight line) to $V=0.1$ (most curved line) in steps $\Delta V=0.1$.

Table 1. Asymptotic dispersion and overall quadratic cost of various phase estimators. For comparison, CRLB is shown. Note that phase prediction of the ML estimation with physical constraint on the inferred value of visibility is superior to the prediction without the constraint.

\begin{tabular}{lll}
\hline Estimator & $\sigma^{2}$ & $\bar{C} \equiv \int \sigma^{2} \mathrm{~d} \bar{\theta}$ \\
\hline NFM & $1 / N$ & $2 \pi / N$ \\
Unconstrained ML & $\left(1+\cos ^{2} 2 \bar{\theta}\right) / 2 N$ & $\frac{3}{2} \pi / N$ \\
Constrained ML & $\approx\left(1+0.5 \cos ^{2} 2 \bar{\theta}\right) / 2 N$ & $\approx \frac{5}{4} \pi / N$ \\
CRLB & $1 / 2 N$ & $\pi / N$ \\
\hline
\end{tabular}

An experimental comparison of the three phase estimations in the asymptotic regime is shown in figure 6 . For comparison, the theoretical values of dispersions given by equations (13) and (14) are also shown. The dispersions were determined using 10000 measured samples with $N=160$ for each value of the true phase shift $\bar{\theta}=k \pi / 16 \mathrm{rad}$, $k=0,1, \ldots, 8$. More than $10^{9}$ weak laser pulses were sent through the interferometer to obtain the figure. Several important conclusions can be drawn from figure 6 .

(1) We can see that the uncertainty of the constrained ML estimation is definitely below the uncertainty of the unconstrained estimation in agreement with our arguments presented in this section. It means that insisting on the physical constraints $\dagger$ of allowed results of estimation or reconstruction procedure is important not only for interpretation reasons, but it also makes the estimation more efficient.

(2) The observed values of dispersion exhibit a systematic error. The additional noise above the theoretical uncertainty is caused by inherent phase fluctuations in the experimental set-up, originating in phase drift and the inaccuracy of adjusting the phase difference, and their magnitude can be estimated from figure 6 as $0.020 \pm 0.003 \mathrm{rad} \neq$. This value is in excellent agreement with the value $0.019 \mathrm{rad}$ obtained by an independent

$\dagger$ Here non-negative definiteness of the intensity.

† Most of this value originates in the inaccuracy of phase difference adjustment. The phase drift between two successive calibrations of the interferometer was negligible.

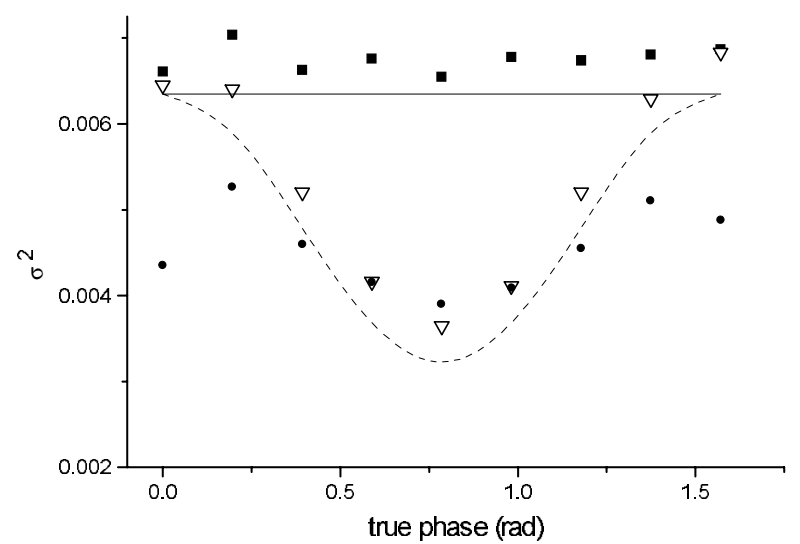

Figure 6. Asymptotic dispersion of the NFM estimator; theory (solid line) and experimentally obtained values (squares).

Asymptotic dispersion of the unconstrained ML estimator; theory (dashed curve) and experimentally obtained values (triangles). Experimentally obtained dispersion of the constrained ML estimation (circles). The corresponding input mean number of photons and the estimated visibility are $N=160$ and $V=99.2 \%$, respectively.

method§. Hence, our statistically motivated evaluation of experimental data can be used for inferring the amount of fluctuations, and therefore it provides an independent and nontrivial way for calibrating an interferometer. Moreover, a slightly different sensitivity of different phase estimators to various parameters of the set-up makes it possible, at least in principle, to distinguish between different sources of noise. This is another interesting feature of the method we propose.

(3) In section 4 we could see that the most distinct difference between semiclassical and fully quantum phase concepts occurs in the regime, where the intrinsic phase uncertainty of light is much larger than phase fluctuations caused by any reasonable imperfections of the experimental set-up. Therefore, though clearly visible in figure 6 , the 'external' phase fluctuations may be completely neglected in figure 2. However, with increasing intensity the (unavoidable) fluctuations become comparable with the intrinsic phase uncertainty, and for even larger $N$ the accuracy of any phase measurement is governed by the external influences rather than by the theoretical limit of the corresponding phase estimation. The statistics of light are then no longer reflected in its phase properties, and different quantum phase concepts become indistinguishable. Not only does this provide another evidence for the fact that the NFM phase concept differs from its ML counterpart only for a narrow range of intensities, as we already stated in section 4 , but it also shows how the operationally defined quantum phase approaches its classical limit.

$\S$ In between two successive calibrations of the interferometer, the phase difference was repeatedly measured with intense light, then the value of the true phase was subtracted, and the standard deviation of the resulting ensemble was calculated. 


\section{Conclusion}

Theoretical and experimental justifications of operational quantum phase concepts have been addressed in this paper. Statistically motivated evaluation of the interferometric setup has been presented. The choice of the optimum phase estimator strongly depends on the researcher's knowledge of the interferometric set-up and on the nature of the signal being detected. Two important cases - the NFM and ML estimators, resulting from the classical and quantum descriptions of the experiment, respectively, have been compared. Differences between both treatments have been measured experimentally and have shown to be statistically significant in the limited range of input intensities. In particular, no difference between the NFM and ML phase predictions have been observed in the regime of a very small number of particles, which is usually considered as the domain of quantum physics. This detailed analysis allows us to quantify the amount of the noise associated with the phase. The lack of knowledge of the parameters of the interferometric set-up has also been considered. In the asymptotic limit of large input intensity, the intrinsically biased ML estimation procedures yield sensible results only provided that all the uncertain parameters of the set-up are estimated together with the unknown phase shift. This can be interpreted in the framework of more complex estimation procedures - the so-called quantum state reconstructions.

\section{Acknowledgments}

We acknowledge support by the TMR Network ERB FMRXCT 96-0057 'Perfect Crystal Neutron Optics' of the European Union, by grant No VS96028 and by research project CEZ:J14/98 'Wave and particle optics' of the Czech Ministry of Education.

\section{Appendix A. Asymptotic dispersion of the Gaussian (NFM) estimator}

To calculate the dispersion of the phase estimator, we need to evaluate the expectation of the sine and cosine functions of the inferred phase, e.g.

$$
\langle\cos \theta\rangle=\sum_{n_{3}, \ldots, n_{6}} \cos \left[\theta\left(n_{3}, n_{4}, n_{5}, n_{6}\right)\right] \prod_{i=3}^{6} P\left(n_{i}\right) .
$$

Here, the inferred phase shift $\theta$ conditioned by the detection $\left\{n_{3}, n_{4}, n_{5}, n_{6}\right\}$ is given by equations (5) and (8) for the NFM and ML estimations, respectively. Now, suppose the interferometer is fed by a strong pulse with $N \gg 1$. Provided the true phase shift $\bar{\theta} \neq k \pi / 4, k \in \mathcal{N}$, we also have $\bar{n}_{i} \gg 1, i=3,4,5,6$, and the Poissonian photocount distribution $P\left(n_{i}\right)$ can be approximated by Gaussian with the same variance, near its peak:

$$
\begin{gathered}
P\left(n_{i}\right)=\frac{\bar{n}_{i}^{n_{i}}}{n_{i} !} \mathrm{e}^{-\bar{n}_{i}} \approx \frac{\mathrm{e}^{-\left(n_{i}-\bar{n}_{i}\right)^{2} / 2 \bar{n}_{i}}}{\sqrt{2 \pi \bar{n}_{i}}}, \\
\bar{n}_{i} \gg 1, \quad n_{i}-\bar{n}_{i} \ll \bar{n}_{i} .
\end{gathered}
$$

Note that although the distribution now becomes symmetric (i.e., the estimation is unbiased for large $N$ ), the noise remains phase sensitive even for high input intensity in contrast with the assumption hidden in the NFM theory (2). Using the Gaussian phase formula (3) in (A1), we obtain the expectation value of the cosine phase function in the following form:

$$
\langle\cos \theta\rangle=\int \frac{n_{34}}{\sqrt{n_{34}^{2}+n_{56}^{2}}} P\left(n_{34}\right) P\left(n_{56}\right) \mathrm{d} n_{34} \mathrm{~d} n_{56},
$$

where $n_{34}=n_{3}-n_{4}, n_{56}=n_{5}-n_{6}$ and we have used the fact that the numbers of counted photons appear in the Gaussian exponential phase estimate (3) only in terms of their differences. The probability distribution of the differences can be calculated from the photocount distributions following the simple rule

$$
P\left(n_{i j}\right)=\iint P\left(n_{i}\right) P\left(n_{j}\right) \delta\left(n_{i}-n_{j}-n_{i j}\right) \mathrm{d} n_{i} \mathrm{~d} n_{j},
$$

where $i j=34,56$. For Gaussian probability distributions we have

$$
P\left(n_{34}\right)=\frac{1}{\sqrt{2 \pi\left(\bar{n}_{3}+\bar{n}_{4}\right)}} \mathrm{e}^{-\frac{\left(n_{34}-\bar{n}_{34}\right)^{2}}{2\left(\bar{n}_{3}+\bar{n}_{4}\right)}},
$$

for example. Since the signal to noise ratio is large in the limit of high intensity, it is legitimate to split the counted numbers of photons (or their differences) into their mean values and small fluctuating parts

$$
n_{i}=\bar{n}_{i}+\Delta_{i}, \quad i=34,56 .
$$

Now we expand the estimated sine and cosine phase functions keeping only the fluctuation-independent term and the second-order terms in the fluctuations. For $\cos \theta$ we get

$$
\begin{aligned}
& \frac{n_{34}}{\sqrt{n_{34}^{2}+n_{56}^{2}}} \approx \frac{\bar{n}_{34}}{\sqrt{\bar{n}_{34}^{2}+\bar{n}_{56}^{2}}}-\frac{3}{2} \frac{\bar{n}_{34} \bar{n}_{56}^{2}}{\left(\bar{n}_{34}^{2}+\bar{n}_{56}^{2}\right)^{5 / 2}} \Delta_{34}^{2} \\
& \quad+\frac{1}{2} \frac{\bar{n}_{34}\left(2 \bar{n}_{56}^{2}-\bar{n}_{34}^{2}\right)^{2}}{\left(\bar{n}_{34}^{2}+\bar{n}_{56}^{2}\right)^{5 / 2}} \Delta_{56}^{2} .
\end{aligned}
$$

The expansion of $\sin \theta$ is obtained exchanging $34 \leftrightarrow 56$. Substituting equations (A6) and (A8) into equation (A4) and using the following relations:

$$
\begin{gathered}
\left\langle\Delta_{34}^{2}\right\rangle=\bar{n}_{3}+\bar{n}_{4}, \quad\left\langle\Delta_{56}^{2}\right\rangle=\bar{n}_{5}+\bar{n}_{6}, \\
\bar{n}_{34}^{2}+\bar{n}_{56}^{2}=N^{2} V^{2}, \quad n_{3}+n_{4}=n_{5}+n_{6}=N,
\end{gathered}
$$

the first set being implied by equation (A6), we arrive at an approximate mean value of the cosine function of the estimated phase

$$
\langle\cos \theta\rangle \approx \cos \bar{\theta}-\frac{1}{2 N V^{2}}\left(\cos ^{3} \bar{\theta}+\cos \bar{\theta} \sin ^{2} \bar{\theta}\right) .
$$

An analogous expression for $\sin \theta$ is obtained exchanging $\cos \bar{\theta} \leftrightarrow \sin \bar{\theta}$ in equation (A11). Finally, using equation (A11) and a similar expression for $\sin \theta$ in the dispersion formula (10) and neglecting terms of the order $1 / N^{2}$, we arrive at the asymptotic dispersion of the Gaussian estimating procedure (13). Since a finite change in the effectiveness of 
the estimation caused by an infinitely small change of the estimated parameter is unphysical, the derived expression for the asymptotic dispersion also holds for the isolated values of the true phase for which our procedure fails.

In the case of ML estimation we can proceed in a completely analogous way. Starting from the expansion of the Poissonian phase estimator (8) in fluctuations of the number of counted photons, we obtain, by a straightforward but a rather lengthy calculation, desired expectation values of the cosine and sine phase functions

$$
\begin{aligned}
& \langle\cos \theta\rangle \approx \cos \bar{\theta}+\sum_{i=3}^{6} C_{i}(\bar{\theta}, V, N) \bar{n}_{i}, \\
& \langle\sin \theta\rangle \approx \sin \bar{\theta}+\sum_{i=3}^{6} S_{i}(\bar{\theta}, V, N) \bar{n}_{i} .
\end{aligned}
$$

Here $C_{i}$ and $S_{i}$ are the coefficients of the terms of the Taylor series, quadratic in corresponding fluctuations $\Delta_{i}$. When an explicit form of the coefficients is substituted into (A12), it is then easy to obtain the asymptotic dispersion (14) of the unconstrained Poissonian ML estimator (8).

\section{Appendix B. Asymptotic dispersion of the single-parameter Poissonian estimator}

As above, we will suppose that detected numbers of photons can be decomposed into their mean values and fluctuating parts, small with respect to the means, as follows; $n_{i}=$ $\bar{n}_{i}+\Delta n_{i}$. Inspection of the Poissonian likelihood function (7) shows that the point $\theta$ is a local maximum of $\mathcal{L}$ if and only if the condition

$$
\frac{\mathrm{d}}{\mathrm{d} \bar{\theta}} \ln [\mathcal{L}(\theta)]=0
$$

holds. Assuming a perfect experimental set-up, $V=1$, the derivative of the log-likelihood function becomes

$$
\begin{aligned}
& \mathcal{L}_{\log }^{\prime}(\bar{\theta})=-n_{3} \frac{\sin \bar{\theta}}{1+\cos \bar{\theta}}+n_{4} \frac{\sin \bar{\theta}}{1-\cos \bar{\theta}}+n_{5} \frac{\cos \bar{\theta}}{1+\sin \bar{\theta}} \\
& \quad-n_{6} \frac{\cos \bar{\theta}}{1-\sin \bar{\theta}},
\end{aligned}
$$

where $\mathcal{L}_{\log }^{\prime} \equiv \mathrm{d} \ln (\mathcal{L}) / \mathrm{d} \bar{\theta}$. Now we make use of the fact that the result of unconstrained ML estimation (8) is not so bad and in particular it lies close to the true global maximum of the likelihood function (7). The purpose is two-fold. First, the estimated phase (8) is a good starting point for finding a root of the expression (B2) with the help of some approximation method; second, it automatically selects the global maximum of $\mathcal{L}$ among all possible roots of equation (B1).

In order to improve our initial guess $\theta_{0}$ :

$$
\theta_{0} \equiv \arg \left\{\mathrm{e}^{\mathrm{i} \theta}\right\}
$$

where $\exp (\mathrm{i} \theta)$ is given by equation (8), we use the Newton method. Since the second derivation of log-likelihood always differs from zero, the algorithm converges quickly and one step of the method is usually enough to find the global maximum with sufficient accuracy. The improved value of the estimated phase shift thus reads

$$
\theta=\theta_{0}-\frac{\mathcal{L}^{\prime}\left(\theta_{0}\right)}{\mathcal{L}^{\prime \prime}\left(\theta_{0}\right)}
$$

Now we expand the sine and cosine phase functions around $\theta_{0}$ keeping terms up to the second order in the correction. The expectation values of the two functions become

$$
\begin{aligned}
& E\left\{\begin{array}{c}
\cos \theta \\
\sin \theta
\end{array}\right\}=E\left\{\begin{array}{c}
\cos \theta_{0} \\
\sin \theta_{0}
\end{array}\right\} \pm \frac{1}{2} E\left\{\begin{array}{cc}
\sin \theta_{0} & \mathcal{L}^{\prime}\left(\theta_{0}\right) \\
\cos \theta_{0} & \overline{\mathcal{L}^{\prime \prime}\left(\theta_{0}\right)}
\end{array}\right\} \\
& -\frac{1}{6} E\left\{\begin{array}{c}
\cos \theta_{0} \\
\sin \theta_{0}
\end{array}\left[\frac{\mathcal{L}^{\prime}\left(\theta_{0}\right)}{\mathcal{L}^{\prime \prime}\left(\theta_{0}\right)}\right]^{2}\right\} \text {. }
\end{aligned}
$$

Finally, using equations (8), (9), (B2) and (B3) in (B5), expanding the result to the second order in fluctuations $\Delta_{i}$, replacing Poissonian photocount distributions by Gaussian with the same variance, carrying out the average, substituting the resulting expectation values of the sine and cosine phase functions into equation (10), expanding the dispersion in $1 / N$ and keeping terms at most linear in $1 / N$, we arrive at the asymptotic dispersion of the one-parameter ML phase estimation in the form

$$
\sigma^{2}=\frac{1}{2 N}+\mathrm{O}\left(\frac{1}{N^{2}}\right) .
$$

The enormous amount of calculation work necessary to obtain this result was carried out with the help of the Maple $\mathrm{V}$ symbolic mathematical language.

\section{References}

[1] Peres A 1998 Quantum Theory: Concepts and Methods (Dordrecht: Kluwer)

[2] Noh J W, Fougères A and Mandel L 1992 Phys. Rev. A 45 424

[3] Noh J W, Fougères A and Mandel L 1993 Phys. Rev. Lett. 71 2579

[4] Řeháček J, Hradil Z, Zawisky M, Pascazio S, Rauch H and Peřina J 1999 Phys. Rev. A 60473

[5] Helstrom C W 1976 Quantum Detection and Estimation Theory (New York: Academic)

[6] Jones K R W 1991 Ann. Phys., NY 207140

[7] Hradil Z, Myška R, Peřina J, Zawisky M, Hasegawa $Y$ and Rauch H 1996 Phys. Rev. Lett. 764295

[8] Zawisky M, Hasegawa Y, Rauch H, Hradil Z, Myška R and Peřina J 1998 J. Phys. A: Math. Gen. 31551

[9] Noh J W, Fougères A and Mandel L 1991 Phys. Rev. Lett. 67 1426

[10] Lane A S, Braunstein S L and Caves C M 1993 Phys. Rev. A 471667

[11] Hradil Z 1997 Phys. Rev. A 55 R1561

[12] Kendall M G and Stuart A 1961 Advanced Theory of Statistics vol 2 (London: Charles Griffin)

[13] Holland M J and Burnett K 1993 Phys. Rev. Lett. 711355

[14] Yurke B, McCall S L and Klauder J R 1986 Phys. Rev. A 33 4033

[15] Walkup J F and Goodman J W 1973 J. Opt. Soc. Am. 63399 